PROCEEDINGS OF THE

AMERICAN MATHEMATICAL SOCIETY

Volume 129, Number 1, Pages 173-179

S 0002-9939(00)05475-7

Article electronically published on June 14, 2000

\title{
A PALEY-WIENER THEOREM \\ FOR THE SPHERICAL LAPLACE TRANSFORM ON CAUSAL SYMMETRIC SPACES OF RANK 1
}

\author{
NILS BYRIAL ANDERSEN AND GESTUR ÓLAFSSON
}

(Communicated by Roe Goodman)

\begin{abstract}
We formulate and prove a topological Paley-Wiener theorem for the normalized spherical Laplace transform defined on the rank 1 causal symmetric spaces $\mathcal{M}=S O_{o}(1, n) / S O_{o}(1, n-1)$, for $n \geq 2$.
\end{abstract}

\section{INTRODUCTION}

The spherical Laplace transform on causal symmetric spaces was introduced in [FHO §8] as a generalization of the spherical Fourier transform on Riemannian symmetric spaces defined by Helgason (see [H1, Chapter 4]). Both transforms can be expressed in terms of (integrating against) spherical functions. It was furthermore shown in [01, §5] that the spherical functions on the Riemannian dual of a causal symmetric space can be written as an expansion in spherical functions on the causal symmetric space. The inversion formula for the spherical Laplace transform easily follows (see [01, §6]).

One of the most important results on the spherical Fourier transform is the (topological) Paley-Wiener theorem (see [H1, Chapter 4, §7] and [H2, Chapter 3, §5] for details) generalizing the classical Paley-Wiener theorem on Euclidean spaces. In this paper we generalize these results to the normalized spherical Laplace transform on causal symmetric spaces $\mathcal{M}$ of rank 1 , thereby partially solving an open problem posed by the second author in [O2, §5].

The paper is divided into two sections: in the first section we recall some results on the spherical Fourier transform on the Riemannian dual $\mathcal{M}^{d}$ of $\mathcal{M}$, and in the second we consider the spherical Laplace transform defined on $\mathcal{M}$. We define the Paley-Wiener space, the supposed image space of spherical Laplace transform, according to the growth and symmetry conditions satisfied by the spherical functions on $\mathcal{M}$. The Paley-Wiener theorem for the normalized spherical Laplace transform follows by using Cauchy's theorem to change the path of integration in the inversion formula and from the Paley-Wiener theorem for the spherical Fourier transform on $\mathcal{M}^{d}$.

Received by the editors December 9, 1998 and, in revised form, March 22, 1999.

2000 Mathematics Subject Classification. Primary 43A85, 22E30; Secondary 43A90, 33C60.

The first author was supported by a postdoc fellowship from the European Commission within the European TMR Network "Harmonic Analysis" 1998-2001 (Contract ERBFMRX-CT97-0159). The second author was supported by LEQSF grant (1996-99)-RD-A-12. 
We have tried to keep notations and proofs to a minimum in order to make the presentation as clear as possible; we refer the reader to [FHO], [HO], O1] and [O2] for more details on spherical functions and the spherical Laplace transform defined on causal symmetric spaces. The spherical Laplace transform in the rank 1 case can be considered as a Laplace-Jacobi transform (see [M] for a detailed analysis of the latter transform) but we note that the Paley-Wiener theorem is new even in the rank one case.

We would like to thank H. Schlichtkrull and J.M. Unterberger for helpful discussion and comments, in particular concerning Lemma 7 and its proof.

\section{NOTATION AND PRELIMINARIES}

In the following we consider the causal symmetric (real hyperbolic) space $\mathcal{M}=$ $G / H$ with $G=S O_{o}(1, n)$ and $H=S O_{o}(1, n-1)$ and its Riemannian dual $\mathcal{M}^{d}=$ $G / K$, where $K=S O_{o}(n)$. Let $\mathfrak{g}$ denote the Lie algebra of $G$ and let $\mathfrak{a}$ be the abelian subalgebra of $\mathfrak{g}$ given by

$$
\mathfrak{a}=\left\{X_{t}=\left(\begin{array}{lll}
0 & 0 & t \\
0 & 0 & 0 \\
t & 0 & 0
\end{array}\right) \mid t \in \mathbb{R}\right\} .
$$

We choose the unique positive root $\alpha \in \mathfrak{a}^{*}$ as $\alpha\left(X_{t}\right)=t$. Let $\mathfrak{a}^{+}=\left\{X_{t} \in \mathfrak{a} \mid t>0\right\}$. We identify the complex dual $\mathfrak{a}_{\mathbb{C}}^{*}$ of $\mathfrak{a}$ with $\mathbb{C}$ via the map $\mathbb{C} \ni z \mapsto z \alpha \in \mathfrak{a}_{\mathbb{C}}$. Let $\mathfrak{n}=\mathfrak{g}_{\alpha}$ and $\overline{\mathfrak{n}}=\mathfrak{g}_{-\alpha}$ denote the positive and negative root space respectively. Let $A=\exp \mathfrak{a}, A^{+}=\exp \mathfrak{a}^{+}, N=\exp \mathfrak{n}$ and $\bar{N}=\exp \overline{\mathfrak{n}}$, where exp is the exponential mapping from $\mathfrak{g}$ to $G$. We also consider the open semigroup $S^{o}=H A^{+} H$ in $G$. Let finally $a_{t}=\exp X_{t} \in A$.

Let $\eta: \mathcal{D}(\mathcal{M}) \rightarrow \mathcal{D}\left(\mathcal{M}^{d}\right)$ denote the Flensted-Jensen isomorphism between the commutative algebras of invariant differential operators on $\mathcal{M}$ and $\mathcal{M}^{d}$ respectively (mapping the Laplace-Beltrami operator $\Delta$ on $\mathcal{M}$ onto the LaplaceBeltrami operator $\Delta^{d}=\eta(\Delta)$ on $\left.\mathcal{M}^{d}\right)$. Let $\Pi(D)$ and $\Pi^{d}\left(D^{d}\right)$ denote the radial part (on $A^{+}$) of $D \in \mathcal{D}(\mathcal{M})$ and $D^{d} \in \mathcal{D}\left(\mathcal{M}^{d}\right)$ respectively. There exists a unique map $C_{c}^{\infty}\left(H \backslash S^{o} / H\right) \ni f \mapsto f^{d} \in C_{c}^{\infty}(K \backslash G / K)$ such that $f_{\mid A^{+}}=f_{\mid A^{+}}^{d}$ and $\Pi(D) f_{\mid A^{+}}=\Pi^{d}(\eta(D)) f_{\mid A^{+}}^{d}$ (see [HO] or [01, §4] for more details).

\section{THE SPHERICAL FOURIER TRANSFORM ON $\mathcal{M}^{d}$}

In this section we recall some well-known definitions and results for the spherical Fourier transform on a Riemannian symmetric space (see e.g. [H1, Chapter 4] and [H2, Chapter 3]).

Let $\lambda \in \mathbb{C}$. Define the Poisson kernel for $\mathcal{M}^{d}$ by

$$
K A N \ni \text { kan }=x \mapsto a^{-(\lambda+\rho)}=: p_{\lambda}^{d}(x),
$$

where $\rho=(n-1) / 2$. The spherical functions on $\mathcal{M}^{d}$ can be written as

$$
\psi_{\lambda}^{d}\left(a_{t}\right)=\int_{K} p_{-\lambda}^{d}\left(a_{t} k\right) d k={ }_{2} F_{1}\left(\frac{1}{2}(\lambda+\rho), \frac{1}{2}(-\lambda+\rho) ; \rho+\frac{1}{2} ;-\sinh ^{2} t\right),
$$

for $a_{t} \in A$. The spherical functions are bi- $K$-invariant and $\Delta^{d} \psi_{\lambda}^{d}=\left(\lambda^{2}-\rho^{2}\right) \psi_{\lambda}^{d}$ for all $\lambda \in \mathbb{C}$. They satisfy the following inequality, for all $\lambda \in \mathbb{C}$ and all $a_{t} \in A$ :

$$
\left|\psi_{\lambda}^{d}\left(a_{t}\right)\right| \leq c(1+|t|) e^{(|\operatorname{Re} \lambda|-\rho)|t|},
$$

for some constant $c$, and they are invariant under sign change, i.e. $\psi_{-\lambda}^{d}=\psi_{\lambda}^{d}$. 
The spherical Fourier transform $\mathcal{F}$ on $\mathcal{M}^{d}$ is defined as

$$
\mathcal{F}(f)(\lambda)=\int_{G} f(x) \psi_{\lambda}^{d}(x) d x=\int_{A^{+}} f(a) \psi_{\lambda}^{d}(a) \delta(a) d a,
$$

for $f \in C_{c}^{\infty}(K \backslash G / K)$, where $\delta\left(a_{t}\right)=\sinh ^{2 \rho} t$. Let

$$
c^{d}(\lambda):=\int_{\bar{N}} p_{\lambda}^{d}(\bar{n}) d \bar{n}=\frac{\Gamma(2 \rho)}{\Gamma(\rho)} \frac{\Gamma(\lambda)}{\Gamma(\lambda+\rho)}
$$

denote the Harish-Chandra $c$-function for $\mathcal{M}^{d}$. We note that $\left|c^{d}(\lambda)\right|^{2}=c^{d}(\lambda) c^{d}(-\lambda)$ for $\lambda \in i \mathbb{R}$. The inversion formula for $\mathcal{F}$ reads (after normalizing $d \lambda$ suitably):

$$
f(x)=\int_{i \mathbb{R}} \mathcal{F}(f)(\lambda) \psi_{-\lambda}^{d}(x)\left|c^{d}(\lambda)\right|^{-2} d \lambda,
$$

for all $f \in C_{c}^{\infty}(K \backslash G / K)$ and $x \in G$.

Let $R>0$. Let $C_{R}^{\infty}(K \backslash G / K):=\left\{f \in C_{c}^{\infty}(K \backslash G / K) \mid f\left(a_{t}\right)=0\right.$ for $\left.t>R\right\}$ and define the Paley-Wiener space $\mathcal{H}_{R}(\mathbb{C})$ as the space of even holomorphic functions $g$ on $\mathbb{C}$ of exponential type $R$, i.e. satisfying the estimate

$$
\sup _{\lambda \in \mathbb{C}} e^{-R|\operatorname{Re} \lambda|}(1+|\lambda|)^{N}|g(\lambda)|<\infty,
$$

for all $N \in \mathbb{N} \cup\{0\}$. Furthermore denote by $\mathcal{H}(\mathbb{C})$ the union of the spaces $\mathcal{H}_{R}(\mathbb{C})$, $R>0$.

Theorem 1 (The Paley-Wiener Theorem). The Fourier transform is a topological linear isomorphism from $C_{c}^{\infty}(K \backslash G / K)$ onto $\mathcal{H}(\mathbb{C})$. More precisely it is a topological linear isomorphism from $C_{R}^{\infty}(K \backslash G / K)$ onto $\mathcal{H}_{R}(\mathbb{C})$ for all $R>0$.

\section{The SPHERICAL LAPLACE TRANSFORM ON $\mathcal{M}$}

We define spherical functions on $\mathcal{M}$ according to O1, Definition 4.1]:

Definition 2. An $H$-bi-invariant continuous function $\varphi: S^{o} \rightarrow \mathcal{C}$ is called a spherical function if there exists a character $\chi$ of $\mathcal{D}(\mathcal{M})$ such that (in the sense of distributions) $D \varphi=\chi(D) \varphi$ for all $D \in \mathcal{D}(\mathcal{M})$.

Define the Poisson kernel for $\mathcal{M}$ (and the open orbit $H A N$ ) by

$$
H A N \ni \text { han }=x \mapsto a^{-(\lambda+\rho)}=: p_{\lambda}(x)
$$

and $p_{\lambda} \equiv 0$ on $G \backslash H A N$. We can construct spherical functions $\varphi_{\lambda}$ as follows:

$$
\varphi_{\lambda}(x):=\int_{H} p_{-\lambda}(x h) d h,
$$

for $x \in S^{o}$, whenever this integral exists (see 01 Theorem 4.10]). Using the calculations in [FHO, §10] we see that the integral converges for $x \in S^{o}$ and $\operatorname{Re} \lambda<$ $1-\rho$ and we get the following explicit formula for $\varphi_{\lambda}$ :

$$
\varphi_{\lambda}\left(a_{t}\right)=c(\lambda)(2 \cosh t)^{\lambda-\rho} F_{1}\left(\frac{1}{2}(-\lambda+\rho), \frac{1}{2}(-\lambda+\rho+1) ; 1-\lambda ; \cosh ^{-2} t\right),
$$

where

$$
c(\lambda):=\int_{\bar{N}} p_{\lambda}(\bar{n}) d \bar{n}=2^{2 \rho-1} \Gamma(\rho) \frac{\Gamma(-\lambda-\rho+1)}{\Gamma(-\lambda+1)} .
$$

We note that $\Delta \varphi_{\lambda}=\left(\lambda^{2}-\rho^{2}\right) \varphi_{\lambda}$ when defined. 
Let $\varphi_{\lambda}^{o}$ denote the normalized spherical function $\varphi_{\lambda}^{o}(x)=c(\lambda)^{-1} \varphi_{\lambda}(x)$. We have the following uniform growth estimate on $\varphi_{\lambda}^{o}$ (due to Helgason (rank one) and Gangolli):

Lemma 3. Fix $\sigma>0$. There exists a constant $c_{\sigma}$ such that

$$
\left|\varphi_{\lambda}^{o}\left(a_{t}\right)\right| \leq c_{\sigma} e^{(\operatorname{Re} \lambda-\rho) t}
$$

for $\operatorname{Re} \lambda \leq 0$ and all $t \in] \sigma, \infty[$.

Sketch of the proof. Consider a formal power series solution to the differential equation $\Pi(\Delta) \varphi_{\lambda}^{o}=\delta(t)^{-1} \frac{\partial}{\partial t}\left(\delta(t) \frac{\partial \varphi_{\lambda}^{o}}{\partial t}\right)=\left(\lambda^{2}-\rho^{2}\right) \varphi_{\lambda}^{o}$ of the form $e^{(\lambda-\rho) t} \sum_{n=0}^{\infty} e^{-n t} \Gamma_{n}(\lambda)$, with $\Gamma_{n}(\lambda)$ to be determined (and $\Gamma_{0} \equiv 1$ ). Substitution into the differential equation gives a recurrence formula that defines $\Gamma_{n}(\lambda)$ uniquely for $\lambda \notin \frac{1}{2} \mathbb{N}$. We can estimate $\Gamma_{n}(\lambda)$ as follows: There exist constants $c, \varkappa>0$ such that

$$
\left|\Gamma_{n}(\lambda)\right|<c(1+n)^{\varkappa},
$$

for $R e \lambda \leq 0$ and all $n \in \mathbb{N}$. The above estimate on $\varphi_{\lambda}^{o}$ follows easily. See [BS, §7-9] for a complete proof in a more general setup.

We define the normalized spherical Laplace transform $\mathcal{L}^{\circ}$ on $\mathcal{M}$ as

$$
\mathcal{L}^{o}(f)(\lambda)=\int_{A^{+}} f(a) \varphi_{\lambda}^{o}(a) \delta(a) d a
$$

for $f \in C_{c}^{\infty}\left(A^{+}\right) \cong C_{c}^{\infty}\left(H \backslash S^{o} / H\right)$ and $\operatorname{Re} \lambda<1-\rho$. From the explicit formula for $\varphi_{\lambda}^{o}$, we see that the function $\lambda \mapsto \mathcal{L}^{o}(f)(\lambda)$ extends to a meromorphic function on $\mathbb{C}$ with at most poles for $\lambda \in \mathbb{N}$.

Let $R>r>0$ and define $C_{r, R}^{\infty}\left(A^{+}\right):=\left\{f \in C_{c}^{\infty}\left(A^{+}\right) \mid f\left(a_{t}\right)=0\right.$ for $0<t<$ $r$ and $t>R\}$. We equip $C_{r, R}^{\infty}\left(A^{+}\right)$with the natural Fréchet space topology and $C_{c}^{\infty}\left(A^{+}\right)$with the inductive limit topology. From Lemma 3 we get the following uniform growth estimate on the normalized spherical Laplace transform acting on $C_{r, R}^{\infty}\left(A^{+}\right)$:

Lemma 4. Let $R>r>0$ and let $N \in \mathbb{N} \cup\{0\}$. There exists a constant $c>0$, only depending on $r$ and $R$, such that, for all $f \in C_{r, R}^{\infty}\left(A^{+}\right)$

$$
\sup _{\operatorname{Re} \lambda \leq 0} e^{-r \operatorname{Re} \lambda}\left(1+|\lambda|^{2}\right)^{N}\left|\mathcal{L}^{o} f(\lambda)\right| \leq c \sum_{n=0}^{N}\left\|\left(\Delta+\rho^{2}\right)^{n} f\right\|_{\infty}<\infty .
$$

Proof. Since $\mathcal{L}^{o}(\Delta f)(\lambda)=\left(\lambda^{2}-\rho^{2}\right) \mathcal{L}^{o}(f)(\lambda)$ and $\lambda^{2}=\lambda^{2}-\rho^{2}+\rho^{2}$, we easily get

$$
\begin{gathered}
\left(1+|\lambda|^{2}\right)^{N}\left|\mathcal{L}^{o}(f)(\lambda)\right|=\sum_{n=0}^{N}\left(\begin{array}{c}
N \\
n
\end{array}\right)|\lambda|^{2 n}\left|\mathcal{L}^{o}(f)(\lambda)\right| \\
=\sum_{n=0}^{N}\left(\begin{array}{c}
N \\
n
\end{array}\right)\left|\mathcal{L}^{o}\left(\left(\Delta+\rho^{2}\right)^{n} f\right)(\lambda)\right| \leq c e^{r \operatorname{Re} \lambda} \sum_{n=0}^{N}\left(\begin{array}{c}
N \\
n
\end{array}\right)\left\|\left(\Delta+\rho^{2}\right)^{n} f\right\|_{\infty},
\end{gathered}
$$

for $\operatorname{Re} \lambda \leq 0$ and all $f \in C_{r, R}^{\infty}\left(A^{+}\right)$, where $c>0$ is a constant only depending on $r$ and $R$.

Using the correspondence between (the radial parts of) invariant differential operators on $\mathcal{M}$ respectively on $\mathcal{M}^{d}$ (see also [01, Theorem 5.9]), we get

$$
\psi_{\lambda}^{d}(a)=c^{d}(\lambda) \varphi_{\lambda}^{o}(a)+c^{d}(-\lambda) \varphi_{-\lambda}^{o}(a),
$$


for $a \in A^{+}$and $\lambda \notin \mathbb{Z} \backslash\{0\}$ (or use [O1, p.63, Eq (17) \& p.64,Eq (22)] and the hypergeometric expressions for the spherical functions), which is the Harish-Chandra expansion formula for $\psi_{\lambda}^{d}$. Let $f \in C_{c}^{\infty}\left(A^{+}\right)$. We see that $\mathcal{L}^{o} f$ satisfies the following functional equation:

$$
c^{d}(\lambda) \mathcal{L}^{o}(f)(\lambda)+c^{d}(-\lambda) \mathcal{L}^{o}(f)(-\lambda)=\mathcal{F}\left(f^{d}\right)(\lambda),
$$

for $\lambda \notin \mathbb{Z} \backslash\{0\}$. The inversion formula for the normalized spherical Laplace transform is now an easy consequence of the inversion formula for the spherical Fourier transform (see also [M, p. 993]):

Theorem 5 (The Inversion Formula). Let $f \in C_{c}^{\infty}\left(A^{+}\right)$. Then

$$
f(a)=2 \int_{i \mathbb{R}} \mathcal{L}^{o}(f)(\lambda) \psi_{-\lambda}^{d}(a) c^{d}(-\lambda)^{-1} d \lambda,
$$

for all $a \in A^{+}$.

All the above suggests the following definition of the Paley-Wiener space, the supposed image space of the normalized spherical Laplace transform:

Definition 6. Let $R>r>0$. We define the Paley-Wiener space $P W_{r, R}(\mathbb{C})$ as the space of meromorphic functions $g$ on $\mathbb{C}$, with at most poles for $\lambda \in \mathbb{N}$, such that

(i)

$$
\sup _{\operatorname{Re} \lambda \leq 0} e^{-r \operatorname{Re} \lambda}(1+|\lambda|)^{N}|g(\lambda)|<\infty,
$$

for all $N \in \mathbb{N} \cup\{0\}$, and

(ii) the $c^{d}$-weighted average $\mathrm{P}^{\text {av }} g(\lambda):=c^{d}(\lambda) g(\lambda)+c^{d}(-\lambda) g(-\lambda)$ extends to a function in $\mathcal{H}_{R}(\mathbb{C})$.

Furthermore denote by $P W(\mathbb{C})$ the union of the spaces $P W_{r, R}(\mathbb{C})$ over all $R>r>$ 0 .

We define a Fréchet space topology on $P W_{r, R}(\mathbb{C})$ by means of the seminorms

$$
\sigma_{r, N}(g)=\sup _{\operatorname{Re} \lambda \leq 0} e^{-r \operatorname{Re} \lambda}(1+|\lambda|)^{N}|g(\lambda)|
$$

and

$$
\sigma_{R, N}(g)=\sup _{\lambda \in \mathbb{C}} e^{-R|\operatorname{Re} \lambda|}(1+|\lambda|)^{N}\left|\mathrm{P}^{\mathrm{av}} g(\lambda)\right| .
$$

We furthermore equip the space $P W(\mathbb{C})$ with the inductive limit topology.

We remark that $\mathrm{P}^{\text {av }} \mathcal{L}^{o}$ acts injectively on $C_{c}^{\infty}\left(A^{+}\right)$, since $\mathrm{P}^{\mathrm{av}} \mathcal{L}^{o}(f)=\mathcal{F}\left(f^{d}\right)=0$ implies $f=f^{d}=0$ on $A^{+}$for any $f \in C_{c}^{\infty}\left(A^{+}\right)$, by the injectivity of the spherical Fourier transform is injective. But we will need that $\mathrm{P}^{\text {av }}$ is injective on $P W(\mathbb{C})$. The following lemma and its proof was communicated to us by H. Schlichtkrull.

Lemma 7. Let $g$ be a meromorphic function on $\mathbb{C}$ that satisfies item (i) of Definition 6. Assume that $P^{a v} g=0$. Then $g=0$.

Proof. Let $g^{1}(\lambda)=g(\lambda) / c^{d}(-\lambda)$. Then $\mathrm{P}^{\mathrm{av}} g(\lambda)=2 c^{d}(\lambda) c^{d}(-\lambda) \operatorname{av} g^{1}(\lambda)$, where

$$
\operatorname{avg} g^{1}(\lambda):=\frac{1}{2}\left[g^{1}(\lambda)+g^{1}(-\lambda)\right]
$$


is the average of $g^{1}$ over the Weyl group \pm 1 . It follows from the assumption $\mathrm{P}^{\text {av }} g=0$ that $\operatorname{av} g^{1}=0$. Let

$$
\gamma(s)=\int_{\mathbb{R}} g^{1}(i \lambda) e^{-i s \lambda} d \lambda, \quad s \in \mathbb{R},
$$

denote the Euclidean Fourier transform of $g^{1}(i \cdot)$. It follows from (i) and [H1. Proposition IV.7.2] that (i) is satisfied by $g^{1}$ as well. In particular, $g^{1}(i \cdot) \in L^{1}(\mathbb{R})$. The condition (i) implies that $g$ is holomorphic in an open set containing $\{z \in$ $\mathbb{C} \mid \operatorname{Re} z \leq 0\}$. Moreover, the standard argument with Cauchy's theorem shows that $\gamma$ is supported on $[r, \infty[$. On the other hand, the average av $\gamma$ of $\gamma$ is the Fourier transform of $\operatorname{avg}^{1}(i \cdot)$, which vanishes; hence av $\gamma$ vanishes as well. The support condition now implies that $\gamma=0$. Since the Euclidean Fourier transform is injective on $L^{1}(\mathbb{R})$, we conclude that $g^{1}$, and therefore also $g$, vanishes.

Theorem 8 (The Paley-Wiener Theorem). The normalized spherical Laplace transform $\mathcal{L}^{o}$ is a topological linear isomorphism from $C_{c}^{\infty}\left(A^{+}\right)$onto $P W(\mathbb{C})$. More precisely it is a topological linear isomorphism from $C_{r, R}^{\infty}\left(A^{+}\right)$onto $P W_{r, R}(\mathbb{C})$ for all $R>r>0$.

Proof. By the Paley-Wiener theorem for the spherical Fourier transform, Lemma 4 and the open mapping theorem for Fréchet spaces, it only remains to show that the normalized spherical Laplace transform maps $C_{r, R}^{\infty}\left(A^{+}\right)$onto $P W_{r, R}(\mathbb{C})$ for all $R>r>0$.

Consider the wave packet $\mathcal{I} g \in C^{\infty}\left(A^{+}\right)$of $g \in P W_{r, R}(\mathbb{C})$ defined by

$$
\mathcal{I} g(a)=2 \int_{i \mathbb{R}} g(\lambda) \psi_{-\lambda}^{d}(a) c^{d}(-\lambda)^{-1} d \lambda,
$$

for $a \in A^{+}$. By Cauchy's theorem we get, for $0<t<r$ and $\mu<0$,

$$
\begin{aligned}
\mathcal{I} g\left(a_{t}\right) & =2 \int_{i \mathbb{R}} g(\lambda) \psi_{-\lambda}^{d}\left(a_{t}\right) c^{d}(-\lambda)^{-1} d \lambda \\
& =2 \int_{i \mathbb{R}} g(\lambda+\mu) \psi_{-\lambda-\mu}^{d}\left(a_{t}\right) c^{d}(-\lambda-\mu)^{-1} d \lambda \\
& \rightarrow 0 \quad \text { for } \quad \mu \rightarrow-\infty,
\end{aligned}
$$

since $\left|\psi_{\lambda}^{d}\left(a_{t}\right) c^{d}(\lambda)^{-1}\right| \leq c(1+|\lambda|)^{\rho}(1+t) e^{(|\operatorname{Re} \lambda|-\rho) t}$ for $\operatorname{Re} \lambda \geq 0$, for some constant $c>0$. An easy calculation shows that

$$
\begin{aligned}
\mathcal{I} g\left(a_{t}\right) & =2 \int_{i \mathbb{R}} g(\lambda) \psi_{-\lambda}\left(a_{t}\right) c^{d}(-\lambda)^{-1} d \lambda \\
& =\sum_{w= \pm 1} \int_{i \mathbb{R}} g(w \lambda) \psi_{-w \lambda}^{d}\left(a_{t}\right) c^{d}(-w \lambda)^{-1} d \lambda \\
& =\sum_{w= \pm 1} \int_{i \mathbb{R}} c^{d}(w \lambda) g(w \lambda) \psi_{-w \lambda}^{d}\left(a_{t}\right)\left|c^{d}(\lambda)\right|^{-2} d \lambda \\
& =\int_{i \mathbb{R}}\left(\sum_{w= \pm 1} c^{d}(w \lambda) g(w \lambda)\right) \psi_{-\lambda}^{d}\left(a_{t}\right)\left|c^{d}(\lambda)\right|^{-2} d \lambda \\
& =\int_{i \mathbb{R}} \mathrm{P}^{\mathrm{av}} g(\lambda) \psi_{-\lambda}^{d}\left(a_{t}\right)\left|c^{d}(\lambda)\right|^{-2} d \lambda,
\end{aligned}
$$


which we recognize as the inverse Fourier transform of $\mathrm{P}^{\text {av }} g \in \mathcal{H}_{R}(\mathbb{C})$; whence $\mathcal{I} g\left(a_{t}\right)=0$ for all $t>R$ by the Paley-Wiener theorem for the spherical Fourier transform on $\mathcal{M}^{d}$.

Since $\mathrm{P}^{\text {av }} \mathcal{L}^{o} f=\mathcal{F} f^{d}$ for all $f \in C_{c}^{\infty}\left(A^{+}\right)$, the above also yields

$$
\mathrm{P}^{\mathrm{av}} \mathcal{L}^{o} \mathcal{I} g=\mathcal{F}(\mathcal{I} g)^{d}=\mathrm{P}^{\mathrm{av}} g
$$

for all $g \in P W(\mathbb{C})$; hence Lemma 7 implies that $\mathcal{L}^{\circ} \mathcal{I} g=g$ for all $g \in P W(\mathbb{C})$ and we conclude that $\mathcal{L}^{o}$ maps $C_{c}^{\infty}\left(A^{+}\right)$onto $P W(\mathbb{C})$.

\section{REFERENCES}

[BS] E. P. van den Ban and H. Schlichtkrull, Expansions for Eisenstein integrals on semisimple symmetric spaces, Ark. Mat. 35 (1997), 59-86. MR 98e:22003

[E] Erdélyi ét al, Higher Transcendental Functions, Volume I, McGraw-Hill, New York, 1973.

[FHO] J. Faraut, J. Hilgert and G. Ólafsson, Spherical functions on ordered symmetric spaces, Ann. Inst. Fourier 44 (1994), 927-965. MR 96a:43012

[H1] S. Helgason, Groups and Geometric Analysis, Academic Press, Orlando, 1984. MR 86c:22017

[H2] S. Helgason, Geometric Analysis on Symmetric Spaces, Mathematical Surveys and Monographs. Vol. 39, American Mathematical Society, Providence, Rhode Island, 1994. MR 96h:43009

[HO] J. Hilgert and G. Ólafsson, Causal Symmetric Spaces, Geometry and Harmonic Analysis, Perspectives in Mathematics, Vol. 18, Academic Press, San Diego, 1997. MR 97m:43006

[M] M. Mizony, Une transformation de Laplace-Jacobi, SIAM J. Math. 14 (1983), 987-1003. MR 85e:44002

[O1] G. Ólafsson, Spherical Functions and Spherical Laplace Transform on Ordered Symmetric Spaces, Preprint (see http://math.lsu.edu/ olafsson/publicat.htm), 1997.

[O2] G. Ólafsson, Open problems in Harmonic Analysis on Causal Symmetric Spaces, Ed: J. Hilgert, J. D. Lawson, K-H Neeb and E. B. Vinberg; Positivity in Lie theory, Open Problems, De Gruyter Expositions in Mathematics, Berlin, 1998, 249-270. MR 99h:43021

Institut de Mathématiques, Analyse Algèbrique, Université Pierre et Marie Curie, Case 82, Tour 46-0, $3^{e}$ Étage, 4, place Jussieu, F-75252 Paris Cedex 05, France

E-mail address: byrial@math.jussieu.fr

Current address: Department of Mathematics, Louisiana State University, Baton Rouge, Louisiana 70803

E-mail address: byrial@math.lsu.edu

Department of Mathematics, Louisiana State University, Baton Rouge, Louisiana 70803

E-mail address: olafsson@math.1su.edu 